\title{
DETC2010/MECH-28499
}

\section{DEVELOPING CUSTOMER PREFERENCES FOR CONCEPT GENERATION \\ BY USING ENGINEERING ONTOLOGIES}

\author{
Dongxing Cao* \\ Mechanical Design \& Application Lab \\ Department of Mechanical Engineering \\ Hebei University of Technology, Tianjin 300130, China
}

Ying Liu

Department of Industry and Systems Engineering The Hong Kong Polytechnic University, H.K.

China

\author{
Karthik Ramani Zhanjun Li Victor Raskin \\ Computational Design Innovation Lab \\ School of Mechanical Engineering \\ Purdue University, West Lafayette, 47907,USA
}

Zhanwei Li

Mechanical Design \& Application Lab

Department of Mechanical Engineering

Hebei University of Technology, Tianjin 300130, China

\section{INTRODUCTION}

In high variety market, the needs of customers can obtain the greatest satisfaction [1]. But, some strategies are also required for attracting the customers' preferences towards concepts of a product. In the past decade, some enterprises as well as researchers have recognized that the customer preference really influences the market activity and often decides the final enterprise strategies [2]. Although the market researchers have paid a close attention for customer preferences, they still lack systematic methods for concurrently developing customer preference modeling [3].

Different people show themselves preferences towards the same product. Psychologists view the preference as an interesting human trait for psychological study, and economists view it as an inaccurate fortunate modeling, only for businesses. As for designers and marketers, the preference is an important issue that can lead to success or failure of product development in the marketplace [4]. Generally speaking, the functions, shape and cost of a product can elicit customers' emotions and inspire their purchasing desires, and also produce a kind of preference by consuming a product from customers. Therefore, designers draw many constraints from customer inputs, and the fact that these constraints are dependent on the contexts in which they are elicited makes them important for the product design process [5-6].

Customer preference is formally defined as a reflection of human inner world for a product. It is the objective of the enterprise developing a product in order to meet the present and future needs of the markets. In most cases, the future needs of the market are not completely uncertain. The

\footnotetext{
* Professor and author of correspondence, Phone: (086) 022-60204935, Fax: (086)010-950507 to 716480, Email: dongxingcao@gmail.com.
} 
customer preference modeling captures the information of a product that provides insight for the future plans, such as functionality, cost, shape, color. If the future needs are as predicted, customer preference modeling allows for faster and effective realization of new products that are marketed. However, if customer preferences are not sought out actively, there may be some loss in initial investment. In addition, the function, shape and cost of a product can elicit customers' emotion and inspire their purchasing aspirations [7]. Thus, product can elicit customers' emotion not only by its appearance, but also by consuming a product. For example, the act of purchasing a cell phone can elicit a strong emotion, and the act of using a cell phone can also elicit a strong emotion. Similarly, by playing with a video game of cell phone we get satisfaction from games and the entertainment value it provides. A large number of current research activities currently focus on emotions elicited from the product appearance. In deed, product appearance is used to indicate its characteristics that people can perceive by looking at, touching, hearing, tasting, and smelling a product, i.e. product shape, texture, taste, color, etc. Some characteristics of emotions elicited by product appearance are illustrated based on a large number of surveys from the statistical viewpoints [8-9].

As a large number of product information are described through using the design documents, such as user requirements, customer dialogs, survey reports, etc. at the same time, the concepts of customer preferences are implicit within these documents [10]. Eliciting and extracting customer preference information is essential to achieve success in new product development. However, there is less research on the customer preference modeling using engineering ontologies. Theory and framework of a prototype that concentrates on customer preference modeling is needed at the earlier stages of product design. We will attempt to build a new model that addresses this issue by including ontology-based information retrieval. The rest of the paper is organized as follows. At first, we review the related work and introduce the research approach and ontology modeling for customer preferences in this context. Secondly, the process of preference semantic extraction is described based on preference knowledge base. Finally, an evaluation and analysis of customer preferences are provided to show the validity of our ontology-based model. And the conclusions and discussions are given.

\section{RELATED WORK}

In the past few decades, customer satisfaction has been widely studied [11] and some methods, such as SCSB, ACSI and NCSB, have been successfully applied to enterprises. Especially in Europe, most of countries have taken some measures to make sure the quality of products to the market. In the long-run, more satisfied customers are an asset of the sector and this asset makes the sector more profitable. For example, a laddering technique is adopted to reveal the deeper meaning of products or services for a customer in order to gain more insight about the perception of the market [12-13]. In addition, conjoint analysis is widely used to measure preferences for different product profiles and build market simulation models. D'Ambrosio and Birmingham emphasize on preference-directed design that is based on an imprecise value function generated from a set of preference statements reflecting the relative importance of each customer attribute
[14]. In general, the customer often shows different preferences relative to the same type products, such as function, shape, color, and costs. The ideas in the mind of the customer are represented by higher level concepts [15]. Also, the customers often do not know what they want and the concepts may be influenced by their browsing and experimenting with their options from new product concepts, or often the opinions of their friends and peers (epionions.com). Customer preference representations that can be described by using textual descriptions and shapes representations can be said to be lower level features. The activity of concept generation by the designers is used to reduce this gap between unconstrained/higher level customer beliefs and constrained/ lower level representations. A flexible representation which the customers may interact with, to indicate their preferences, can be modeled using new representations that combine shape and ontologies. The results of the customer interaction by search, relevance feedback and modifications can provide valuable input to the design activity and even group the customers into different categories or classes for further concept generation. Constrained/ lower level features are highly structured, such as final reports, transaction data, geometric shapes, CAD drawing, which we depict as lower level features. At the same time, unconstrained/high level concepts are unstructured, fragmented documents, such as interviews, design logbooks, which they are viewed as higher level concepts [16].

Low-level features, such as shape, size, number of entities, are easily identified by humans. The shape can be specific from geometric point of view or it can be a perceived feature. They have considerable influence on customer purchase decisions [17]. In general, the shape can be easily represented in the hierarchy. This hierarchical representation describes the main shape categories that can be identified [18]. Also it can be indexed in the shape repository. The varied shape information is described by shape representations that can allow us to search the knowledge represented in the shape repository [19]. Some important properties of shape description are also described by using an associated textual description. The Shape type hierarchy captures information regarding the type of shapes that can be processed by a shape semantic description.

Ontology can be not only expressed in a logic-based form, but also made detailed, accurate, consistent and can help arrive at meaningful explanations among the concepts and relations [10]. Moreover, domain ontologies logical form is appropriate for a uniform representation. This description can be used as representing the complex knowledge of the product development domain. In addition, in order to generate concepts, its reasoning capability should be as powerful as possible. Gruber presented the first attempt to consolidate experience gained in developing ontologies [20]. It can be summarized as five ontologies design criteria: coherence, extensibility, minimal ontological commitment, and minimal encoding bias. Uschold and King developed enterprise ontology for enterprise modeling processes [21]. Their development method includes four activities: purpose identification, ontology building, evaluation, and documentation. They also proposed three strategies for identifying the concepts in the ontologies: top-down, bottomup, and middle-out. Gruninger and Fox proposed an ontology 
design and evaluation method while developing the TOVE (Toronto Virtual Enterprise) project ontology [22]. It uses a set of natural language questions, called competency questions, to determine the scope of the ontology and to extract the main concepts of the ontology as well. Their major focus, however, is to build the first-order logical model representation of the ontology. On the other hand, ontology preferences still depend on customer emotions because they are related to customer personality, that is, different people experience different emotions towards the same product. Therefore, one person may experience different emotions towards a specific product at different times. Because products have themselves personalities, different objects or shape features can elicit different emotions [7]. General shape ontology captures shape related metadata and constitutes an ontology-driven evolution of the metadata in the shape repository. A high level hierarchical relationship of the ontology describes the main concepts of shape ontology, which includes shape program, shape repository, shape concepts, and so on [23]. Shape program contains program rules and semantic structures. They can be extracted from the text information. Shape repository stores shape semantic information and structural information. File information can be used to describe shape concepts which capture some information regarding a product or shape associated with the various shape models stored in the repository. The concepts of shape classification can help us clusters of shapes that share some common features [19]. Usually, several elements of a shape that differ in some key feature and are placed in a group so that they can be examined together. File information can store information related to a shape, such as the size, material, color, etc. The shape representation is the central concept in the ontology and encapsulates information that is inherent to the shape model itself. It also constitutes a fundamental concept which is extended by concepts defined in the domain ontologies.

\section{METHOLOGY}

\section{Overview}

The process of developing customer preference ontologies are getting to know the relationships among ontology concepts. Designers should extract some useful information from documents in order to carry out design tasks. As the input contents from customers are disorderly and unstructured documents, sometimes most of them are qualitatively described, such as user requirements, survey reports, transaction data and customer dialogs. They need analysis and transformation into formal and actionable structures. The volume of content to be processed is a barrier that good ontological analysis can overcome. The transformation operation is to combine qualitative with quantitative aspects. Qualitative transformation characterizes some design information with disordered arrangements in an abstract manner. The results are directed to enable designers to make a revision to improve concept description. On the contrary, quantitative transformation provides a canonical document description [25]. They allow designers to easily understand, evaluate and reuse previous design information.

Fig.1 presents the overall system infrastructure of interactions between engineering ontologies for different functional modules. The framework comprises of five portions: transformation, ontological acquisition, concept recognition, concept indexing, query processing, and empirical study. In general, most of the existing information is disorderly and unsystematic. They need designers' analysis and abstraction in order to gain utility. The original documents from customers, such as survey reports, transaction data, and customer dialogs should be filtered. A normal design information text or document is used to extract preference information after transformation [26]. Automatically extracting semantics from the normalized document requires recognizing the domain knowledge as well as the semantic structures of the text. Linguistic knowledge and semantic rules are needed to fulfill preference semantic extraction.

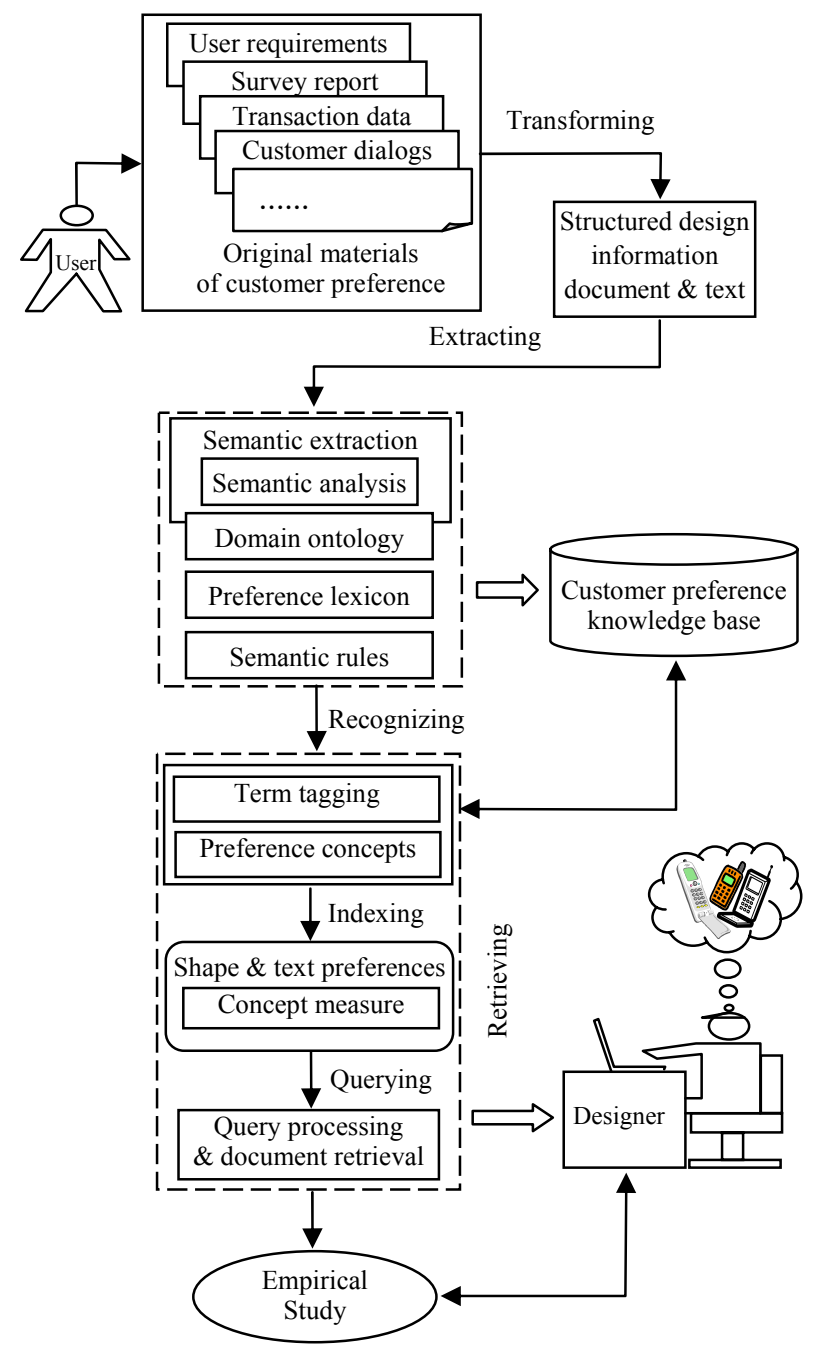

Figure 1. Infrastructure of system

First of all, the original material documents from customers, such as user requirements, survey reports, transaction data, etc. are acquired and then transformed into design information texts or documents in which some unstructured information should be preprocessed by adopting an effective approach. Second, some terms and concepts can be extracted from preference semantic structures, such as noun phrases, verb phrases, adverb phrases and adjective phrases. These can be represented by using an ontology-based design semantic analysis and information extraction. The taxonomy 
of preference is further classified to acquire the relationships between two concepts. The specific thesauri or lexicons are built to capture preference concepts from the preference knowledge base. The ontology expression and preference semantics extraction process are described, and the process of semantic extraction is based on a shallow natural language processing (NLP) algorithm and the domain ontology. Next, the preference ontology concepts are acquired after the extraction process. The extraction algorithm and the preference metrics are described and are used for preference ontology modeling. Finally, an empirical study for design preference extraction is introduced and three group queries are processed. A prototype system interface is provided to aid the process of preference information retrieval in order to capture and generate the concepts of customer preferences

\section{Process of six-stage development}

Conducting a process in several stages develops customer preference ontologies. These stages have a certain hierarchical structural relationship. Different stages have themselves research methods and properties shown in Fig. 2. The process of preference ontology generation with six-stages is described as follows.

(1) Transforming informal documents into the formal knowledge for system input, in which these informal design specification includes user requirements, survey reports, transaction data, designers' logs, patent files, customers' dialogue, and so on;

(2) Determining the preference concept ontologies and their granularities through extracting the words and terms of documents, in which a concept maybe described. The terms may consist of several words, and may have different weights among concepts;

(3) Identifying the root concept of customer preference ontology in the taxonomy, in which the root concept is parent-node of all sub-concepts, and a product may have a number of the root concepts;

(4) Generating the preference ontology concept taxonomies corresponding to each root concept from the previous stage, and according to different product properties each root concept may have different taxonomies depending on product classifications;

(5) Building a thesaurus of preference knowledge to realize preference ontology information for the integrated taxonomy, in which the thesauri include different word morphs, abbreviations, acronyms, and synonyms of the word/phrase. Also, some noun phrases, verb phrases, adverb phrases, and prepositional phrases can be extracted as preference terms.

(6) Evaluating the accuracy of preference ontology generation by comparing the retrieval performance of the ontology-based and keyword-based search, and the validity of concept generation through using case studies.

In the process of system realization, resource description framework (RDF: http://www.w3.org) is used to describe concept ontologies. Their taxonomies are represented in the light of preference attributes and the ontological construction is assisted by the protégé tool (http://protégé. stanford.edu). Each of these stages is companied the process of evaluation and has a corresponding output.

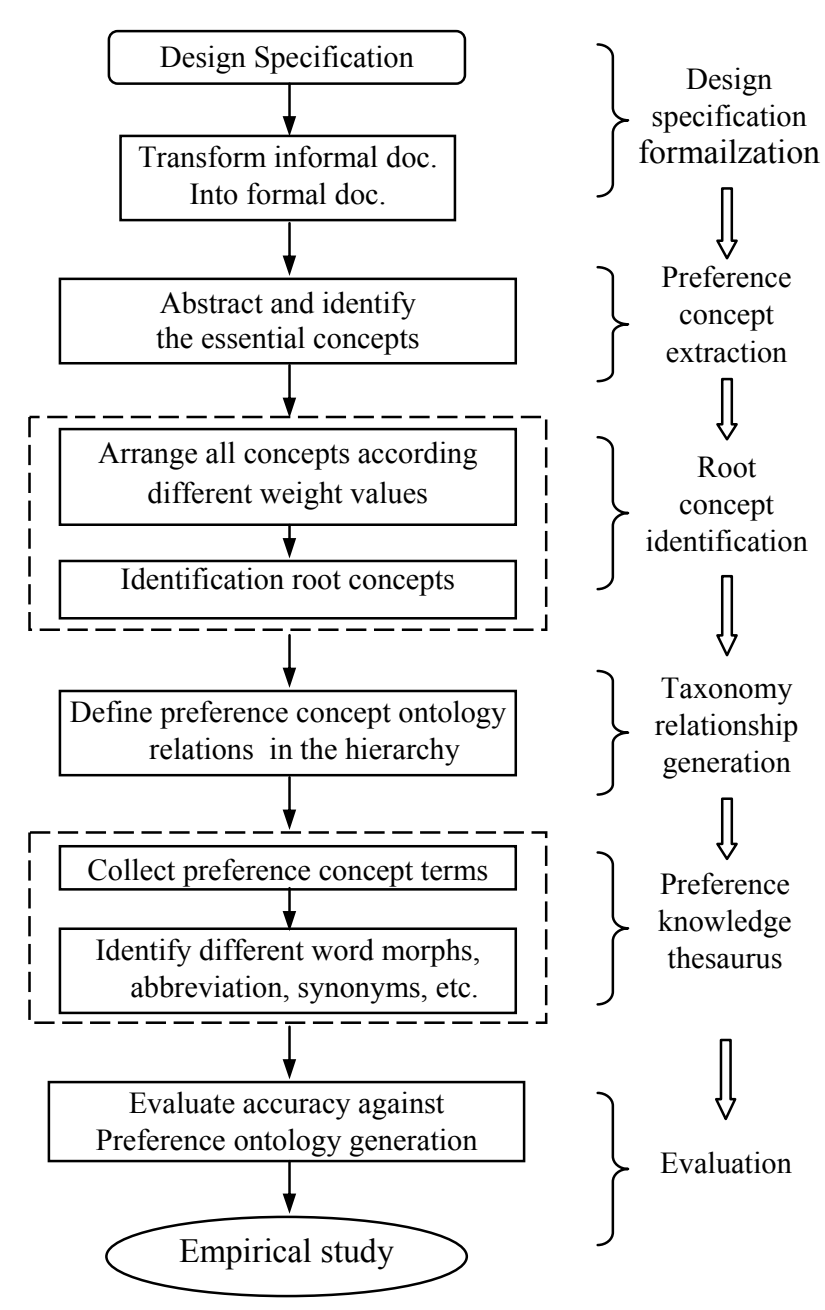

Figure 2. Process of six-stage ontology

\section{PREFERENCE ONTOLOGY GENERATION}

\section{Design Specification Formalization}

Design specification provides information input of design, it includes the related design information documents, such as users' requirements, survey reports, transaction data, designers' logs, patent files, customers' dialogues, and so on. At the beginning of design, we should investigate what types of information are required by engineers and what domainspecific issues are documented during the product development process. The results of these investigations are categorized and used to determine the concept themes of customer preference ontology. For example, they perhaps include component shapes, product assemblies, functionalities and properties of the devices, assembly features, design requirements, manufacturing processes, material selections, environmental aspects, and so on. As most of the knowledge from the customers is unstructured and informal, and it needs to be organized by adopting an effective expression that computers can easily understand and acquire. These expressions are called as knowledge worksheets. They are the format templates which can be used to express design 
knowledge and the related information, and they are viewed as an independent of ontology engineering tools or formal language descriptions. In general, there are two forms of knowledge expressions: domain knowledge and reasoning knowledge. Domain knowledge is about the related specific product knowledge that is obtained from some professional domains. Reasoning knowledge is about how to use the domain knowledge to solve problems and achieve prospective goals. It is often in the form of the rules that can help achieve the goals in a different domain. Domain and reasoning knowledge are generally included in the following two forms:

- The purpose of the preference ontological acquisition, including its intended usages, scenarios, customers, etc. its formal level will depend on the domains of research and using the terms and their meanings.

- The scope of the preference ontologies, including a group of terms to be represented, characteristics and granularities. They will be classified into formal, semi-formal, informal ontologies, depending on the terms and their meanings whether they codify in a natural language and a rigorous formal language.

Formal specification of the documents depends on if we use natural language, symbol description and effective classification. In fact, in this paper, we collect the set of preference concepts by using terms and they should be included in the ontology. It is also advisable to group concepts in tree structures. The use of these intermediate representations will allow us not only to verify, at the earliest possible stage, relevant terms missed and to include them in the specification document, but also to remove terms that are synonyms and not relevant in our ontology anymore. Fig. 3 gives the ontology requirement specification of cell phone. The goal of our formalization is to guarantee the conciseness and completeness of the ontology specification documents.

\section{Domain: electronics \\ Data: Feb. $5^{\text {th }} 2010$ \\ Carrier: AT\&T, Cingular \\ Brand: LG \\ Purpose: building preference ontology concepts of cell phone, including its function, performance, shape, color, manufacturing, environment, etc. \\ Formal level: semi-formal \\ Scope: using preference terms constructs preference concept, these preference terms include domain knowledge and rules, common verbs, nouns, adjective and adv, etc. \\ Sources of knowledge: cell phone handbook, Website}

\section{Figure 3. Ontology requirement specification of cell phone}

If we want to obtain the total completeness of the ontology specification document we should guarantee that it is developed based on scientific principles. It must have the following three properties.

- Conciseness, that is, every concept is relevant, and there are no duplicated or irrelevant concepts and corresponding domain ontologies. For example, "COMMUNICATION" concept of cell phone has a relevance to "TEXT MESSAGE" concept.
- Completeness, which is related with the coverage of the concepts, the stopover problem and level of granularity of every concept. For example, "TEXT MESSAGE" is a subconcept of the "COMMUNICATION" concept.

- Consistency, that refers to all concepts and their corresponding meanings in the domain ontology. At the same time, every concept has its corresponding scales to show its importance in document.

Few domain-independent methodologies and expressions for building preference ontologies have been reported until now. Uschold proposed a methontology that is the most representative process of building ontology to acquire a significant amount of knowledge [21]. It always starts from domain knowledge acquisition. However, Grüninger and Fox's methodology presented coding in a formal language and proposed expressing the idea as a set of intermediate representations [22]. Then the methontology uses translators to generate the ontology.

\section{Identifying the Root Concepts}

At the beginning of the engineering design, the main task is to identify what concepts should be contained within a preference ontology for a specific product. This will include two aspects: (1) identify a specified application that an ontology is to be used for, for example, using for cell phone design, and (2) understanding the root concepts of the taxonomies that can be used to describe product design. The proposed method describes the elicitation of the root concepts from potential customers of the ontology, for example, we can obtain the information of the root concepts through surveying, interviewing or observations of product designers.

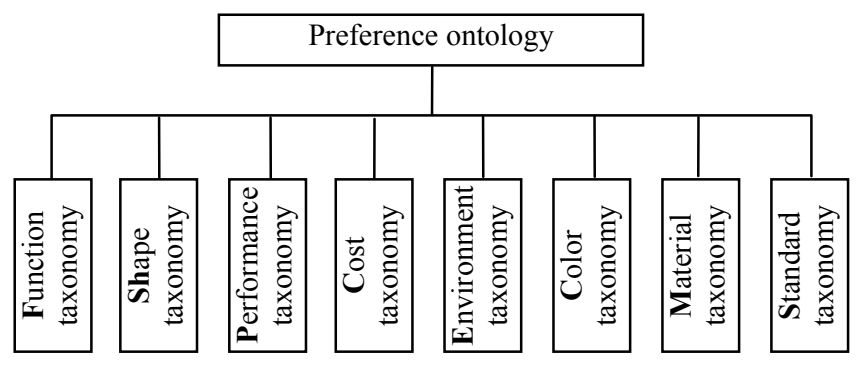

Figure 4. Root concept of preference ontology

Tree structure of ontology concepts can provide an effective approach to indexing terms/concepts which can be used to match with customer requests. However, the taxonomy acquisition of customer preferences of different products is of a certain subjectivity. Their generation is done by brainstorming, by interviewing, or dialogs with customers. Preference ontological acquisition and generation use the same methodology. Fig.4 presents the root-concept taxonomy of customer preference ontology, which comes from cell phone handbooks or knowledge resources. For example, cell phone handbooks often classify engineering components which can be clustered into an ontology model as concepts and their taxonomies in the hierarchy. Each component is described in detail, including its attributes, such as material, physical, geometric and functional properties which can easily be 
identified and mapped to ontologies as well as corresponding relationships.

Customer preference ontology includes concepts, taxonomies and relationships. Each taxonomical concept is acquired from various engineering knowledge resources. We can adopt terms or phrases to describe the concepts of the taxonomy as well as their relationships with other concepts. For example, multimedia belongs to Function taxonomy of cell phone. We can represent as F-MULTIMEDIA, where the prefix of each concept represents its taxonomy which this concept belongs to. Therefore, the relationships are structured between concepts across taxonomies. For example, has feature (COL-SIVER, SH-KITTY-PHONE), in which COL-SIVER stands for a color concept in the color taxonomy, SH-KITTY-PHONE represents a shape concept in the shape taxonomy $[10,27]$. Tab.1 lists customer preference ontological concepts and their relationship classifications of a cell phone.

At present, we have collected 10 taxonomies, 240 concepts and 7 types of relationships in the customer preference ontology. The standard worksheets are developed to easily acquire preference ontology and lexicon. At the same time,

Table 1. Classification of the relationships

\begin{tabular}{|c|c|c|}
\hline Relationship & Concepts & Definition \\
\hline is_a & F-VOICE/ F-MP3 & Parent-son relation \\
\hline has_part & $\begin{array}{l}\text { E-HEALTH RISK/ } \\
\text { E-CYCLE } \\
\end{array}$ & $\begin{array}{c}\text { Part to whole } \\
\text { relation }\end{array}$ \\
\hline has_function & $\begin{array}{c}\text { F-VOICE/ P-LONG } \\
\text { TALK }\end{array}$ & $\begin{array}{l}\text { Refer to the } \\
\text { connection between } \\
\text { two concepts }\end{array}$ \\
\hline use_material & $\begin{array}{c}\text { COS-LOW END } \\
\text { PHONE/ M-METAL }\end{array}$ & $\begin{array}{c}\text { The type of } \\
\text { materials }\end{array}$ \\
\hline has_property & $\begin{array}{c}\text { SH-FLIP PHONE/ M- } \\
\text { STEELLESS }\end{array}$ & $\begin{array}{l}\text { Physical attribute/ } \\
\text { geometric attribute }\end{array}$ \\
\hline has_feature & $\begin{array}{c}\text { COL-SIVER/ SH- } \\
\text { KITTY PHONE }\end{array}$ & Geometric shape \\
\hline has_standard & F-3G/ ST-NETWORK & $\begin{array}{c}\text { Domain specific } \\
\text { standards }\end{array}$ \\
\hline
\end{tabular}

they can be automatically uploaded with the required data into the Protégé editor (http://protege.stanford.edu/) [28]. Therefore, the proposed customer preference ontological concepts can be also presented by using Protégé 3.1, which is one of the most widely used ontology editor. Protégé provides a visual tool for preference ontology editing, including concept, taxonomy, and relationship building as well as preference ontology visualization.

\section{Determining Preference Ontology Concepts}

We identify what taxonomies should be contained within the preference ontology for engineering design. A hypothesis is developed based on an understanding of the design process; an understanding of why designers search for preference information. A test is conducted with a group of customers to test the hypothesis and the designers are also asked to describe the process of design of a cell phone. The methodology describes the elicitation of the preference concepts from the potential customers of the ontology, for example, customer interviews and observations are employed to elicit ontology generation. The application of the preference ontology is for the purpose of indexing design knowledge, and therefore the taxonomies should be considered how designers would wish to index and retrieve knowledge. Hence the concepts that form the root concepts of the ontology and are used for indexing documents are elicited from interviews with the designers. An effective approach can be used to index knowledge that has been captured within a system, including documents that are both structured and unstructured, knowledge such as design guidelines, or lessons learned that have been captured. A set of interviews is conducted to test the hypothesis and the codification of the interviews is then evaluated using coder reliability to establish concept validity. We develop a comprehensive and representative approach to ensuring the final description of product characteristics. In Fig. 5 we have shown the taxonomy of cell phone Function-concepts. Therefore, a set of concepts can be used as the definition of a product. At first, the concepts of customer preferences based on the collection of an extensive pool of customer descriptions

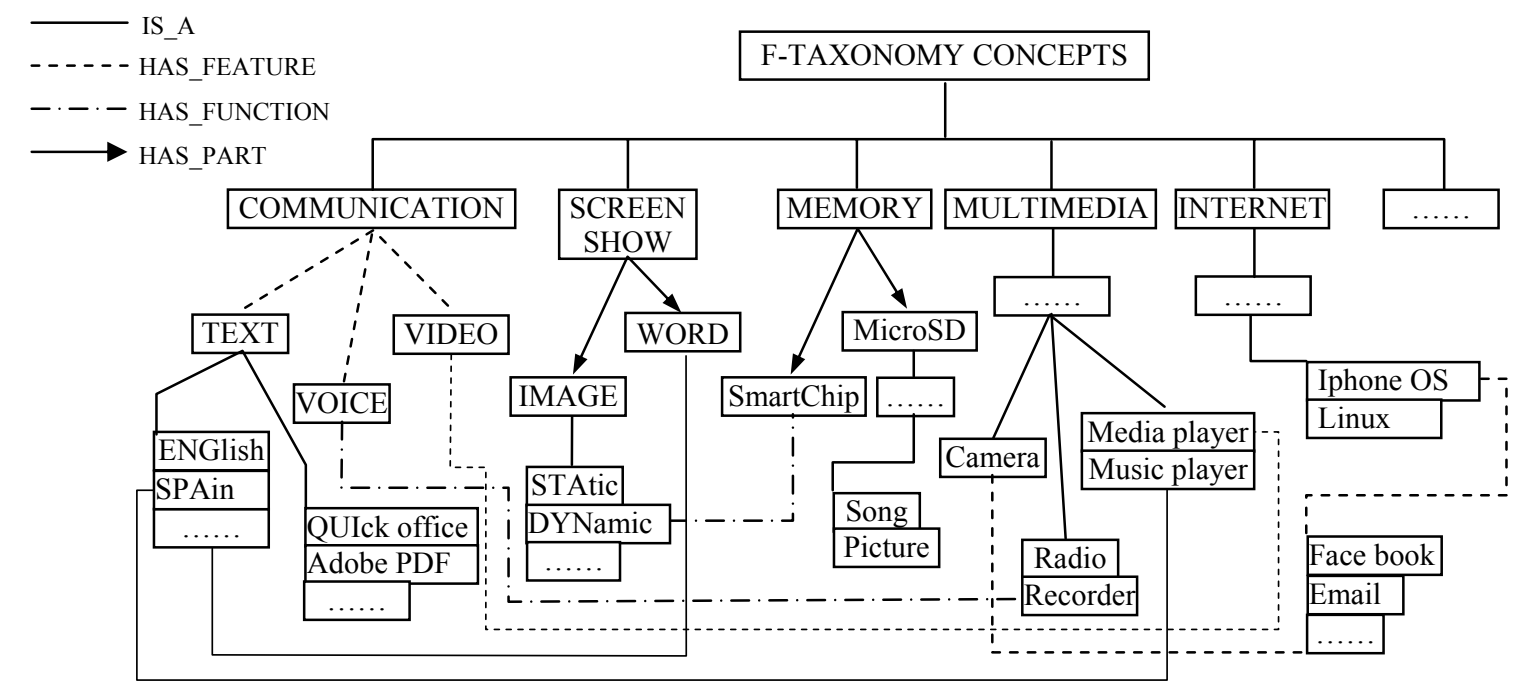

Figure 5. Taxonomy of cell phone F-concepts 
are developed towards a specific product. To ensure that the pool of descriptions is complete, we also collect customer descriptions in a qualitative customer study. For this study, we will randomly select some comments about cell phones from the Website. Accordingly, this allows us to obtain some descriptions of cell phone characteristics that different customers talk about. To enhance the generalization of the resulting scale, we select a variety of cell phone categories that have different values from the customer viewpoints [20].

All 240 concepts are collected from customer descriptions of cell phone, in which 1120 words are formulated in single adjectives. Adjectives are traditionally seen as the primary terms of concepts and are frequently used as items in preference concept scales. Single adjectives are preferred over adverb to representing preference meanings, because when using the latter the meaning of the preference may differ depending on the combination of the adjectives. For example, 'good' is a kind of preference praise, further 'very good' becomes better in praise.

\section{Generating Concept Taxonomies and Relations}

Semantic rules may be used to link preference words and terms together to build the preference concepts to aid expression in design information. In general, the concept consists of some terms by using a fit semantic rule. Term is represented by some words, such as noun, verb, adjective, adv, and so on. XML is a kind of web language and employed to represent data that is interpretable by a computer rather than a human. It is composed of syntax and schema. The syntax defines a set of principles and rules that govern how a concept should be built. The schema defines what the tags mean. XML is extensible as it allows users to define any custom schema, or vocabulary. Resource description framework (RDF) is viewed as a basic component of expressing semantic web. It takes advantage of XML-based syntax, but unlike prescriptive XML schema, RDF schema is descriptive. That is, instead of defining what information can be captured, RDF further enriches the description of the data. Their relationships are described through sets of RDF triples, which each include a subject, a predicate and an object. These triples can be conceptually thought of as similar to the subject, verb and object of a sentence as shown in Fig 6 .

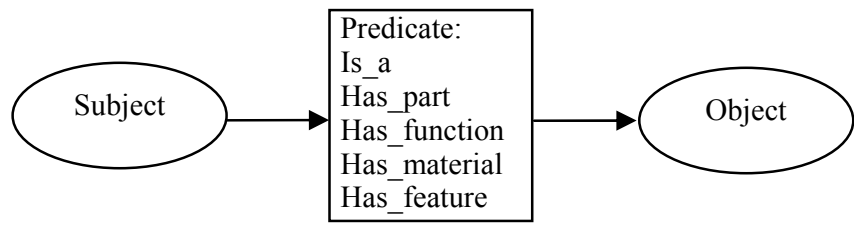

Figure 6. RDF triples

In order to speed up the construction of our preference ontology, we should consider reuse of definitions already built into other ontologies instead of starting from scratch. In this case, we propose the following:

1. We insert meta-ontologies to select those that better fit our conceptualization. The goal is to guarantee that the sets of new and reused definitions are based upon the same set of basic terms. If existing meta-ontologies are not appropriate for our ontology. We should start the definition and implementation of a new meta-ontology in a formal language.

2. Whether or not we reuse existing meta-ontologies, the next step is to find out which libraries of ontologies provide definitions of terms whose semantics and implementation is coherent with the terms identified in our conceptualization. Once we have chosen the most appropriate terms, we should check the existence to transform definitions into our target language.

As a result of concept generation, some terms should be indexed and concepts should be included in a given ontology. In this case, we should justfy the need for the concept definition and their relationships as well as the extended concepts and their relationships. Fig. 7 presents the taxonomy of the cell phone concepts and their extended concepts by using ontology editor Protégé 3.1[28-29]. Here we propose the development of an integration document, and extract some concepts and the name of terms whose definition is going to be used in the preference concept ontology.

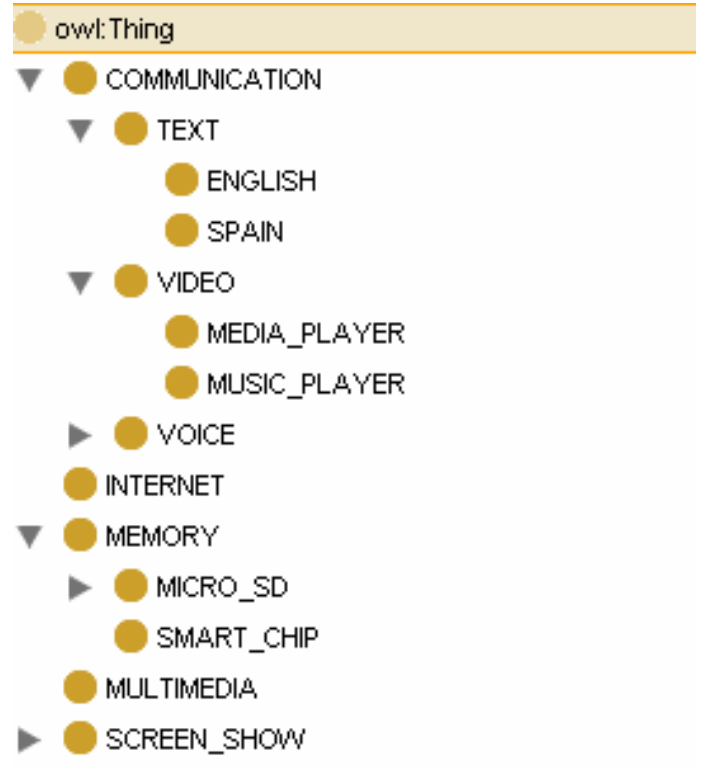

Figure 7. Taxonomy of concepts

\section{Constructing Thesaurus}

It is important to bear in mind that knowledge acquisition is an independent process for preference ontology development. However, it is coincident with other activities, for example, most of the acquisition is done simultaneously with the requirements specification phase, and decreases as the preference ontology development process moves forward. In addition, expert, books, handbooks, figures, tables and even other ontologies are sources of knowledge from which the knowledge can be elucidated and used in conjunction with other techniques such as brainstorming, interviews, formal and informal analysis of texts, and knowledge acquisition tools. For example, if you have no clear idea of the strategy to build an ontology: brainstorming techniques, informal interviews with experts, and inspecting similar ontologies will allow you to elaborate a first glossary with terms potentially relevant. To 
refine the list of terms and their meanings, formal and informal analysis of text techniques in books and handbooks in conjunction with structured and un-structured interviews with experts can be used to include or remove terms in the glossary. Interviews with experts can help to build concepts classifications trees and to contrast them against figures given in books. The techniques we used in the knowledge acquisition phase of the preference ontology includes as follows.

- Un-structured interviews with experts, to inquire some related design problems and to build a preliminary draft the requirements specification document.

- Structured interviews with experts, to get specific and detailed knowledge about concepts, their properties and relationships, to evaluate the conceptual model.

- Informal text analysis, to study the main concepts given in books and handbooks. This study enables us to abstract some intermediate representations of the conceptualization.

- Formal text analysis, to identify the structures to be detected and the kind of knowledge contributed by each concept, attribute, value, and relationships.

On the basis of above descriptions, we structure the domain knowledge in a conceptual model that describes the problem and its solution in terms of the domain vocabulary identified in the ontology specification activity. The first thing to do is to build a complete Preference Concept (PC), in which concepts include terms, instances, verbs and properties. So, the PC identifies and gathers all the useful and potentially usable domain knowledge and its meanings. If you made a good specification document, many concepts will have been identified in that document. Others will be identified as the ontology construction process advances. Then, these new concepts must be included in the PC.

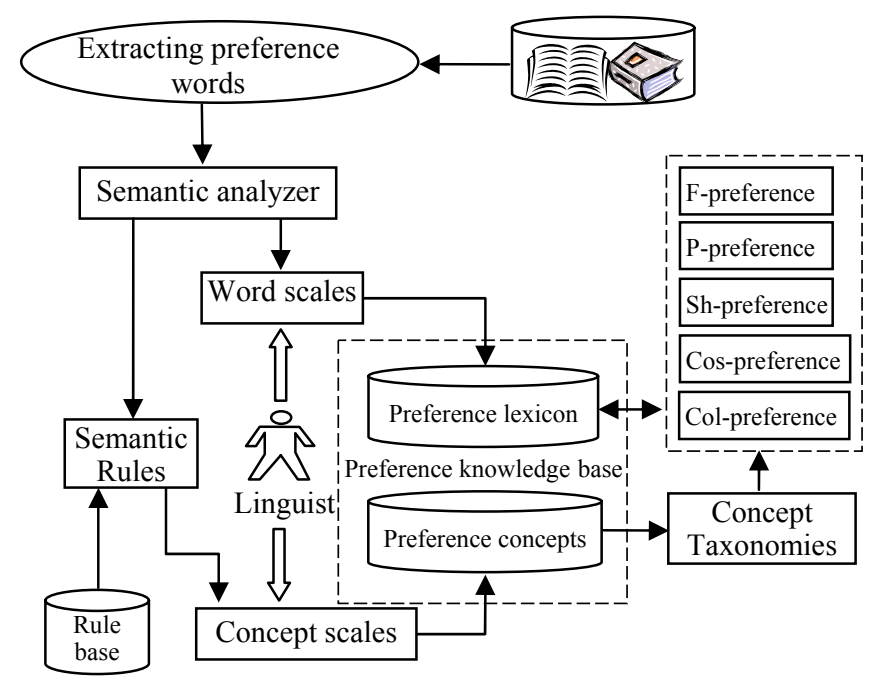

Figure 8. Knowledge base acquisition

Once we have almost completed the PC, it needs to group concepts as terms and verbs. Each set of terms/verbs would include terms/verbs that are closely related to other terms/verbs inside the same group as opposed to other groups. In fact, for each set of related terms and related verbs, a terms classification tree and a verbs diagram is built. After they have been built, we can split the ontology development process into different, but related terms. Those related concepts should follow the guidelines as presented by G6mez-Ptrez [30]. Figure 8 summarizes the process of preference knowledge base construction. At first, extract some words from documents and dictionaries. These words can be classified as different taxonomies based on the semantic description. A semantic analyzer is built to separate these words into different taxonomies. Some independent words will be scaled based on linguistic viewpoints. On the other hand, semantic rules are built to compose the concepts, in which the rule base is crucial and consists of concepts. Also according to the viewpoints of linguist, some concepts should be marked weights to fit their importance. The taggeded words and concepts are put into preference knowledge base. In addition, we need to classify concept taxonomies, as for the case study, we can give different preference concepts, such as function preference, performance preference, cost preference, and so on.

\section{EVALUATION AND ANALYSIS}

\section{System Evaluation}

A framework for evaluating preference knowledge has been developed to verify the validity of the methodology. An effective measure is taken to evaluate a technical judgment of the ontologies, their use environment and documentation with respect to a frame of reference in different stages. Evaluation subsumes the fit concepts and verification refers to the technical process that guarantees the correctness of building the ontology, its associated environments, and documentation with respect to a frame of reference. Validation guarantees that the ontologies, the environment and documentation should correspond to the system that they are supposed to work. Based on the experience of verifying concept ontologies, how to look for incompleteness, inconsistencies and redundancies have been presented in [30]

In general, interviews are conducted to evaluate the taxonomy. These group interviews are used to identify the root concepts. Inquiries are implemented to ensure that the evaluation is taken place after any questions to avoid biasing the answers. The taxonomy is constructed by the designers in advance [31].

Since every word has different contributions to describe the contents of the documents, it is necessary to select only more informative words and to assign relative weights to the importance of each word by considering how informative it is compared to the others. As for the weights of concepts in a document, they are assigned by their frequencies within the documents multiplied by the inverse document frequency which allows distinguishing the few documents. In order to take into account different lengths of the documents, normalizations are performed. A cosine simliarity is used for measuring the similarity distance between the words in the documents and in the taxonomy terms. The two most common problems are known as polysemy and synonymy. Polysemy is caused when a word is used for multiple meanings depending on contexts. For example, several attempts have been made for disambiguating the polysemy and one approach relies on the combinations of words for identifying appropriate meanings of each word. For example, picture appears in function taxonomy concepts "STATIC-PICTURE" and "DYNAMIC-PICTURE" 
because both concepts have the same lexical term picture. Synonymy means that two syntactically different words are semantically interchangeable, for example, "TRANSMIT" and "TRANSFER." These ambiguities are direct reasons for the lower concept retrieval precision. For example, if customers like the price of about $\$ 80$ with volume $80 \times 40 \times 10 \mathrm{~cm}^{3}$ for a cell phone. The two numbers " 80 " often appear ambiguous. In preference ontology concepts, we have divided customer preferences into different classifications. By marking different taxonomical signs during tagging terms, such as, COSMIDDLE PRICE EIGHTY and SH-SIZE EIGHTY, we can distinguish them [27].

The large number of files, including Doc, HTML, XML, can be used as the output data of concepts from the ontology. In addition, appropriate concepts can also be manually specified for users. An algorithm can be developed to compute the similarities between two ontological concepts by integrating node-based and edge-based approach and then rank them according to the obtained similarity values [27]. Users can access and query the system from the Internet, while domain designers can manipulate the document formalization and ontology building process.

\section{Result Analysis}

We collected a total of 45 queries from the five different groups, among which 3 queries were eliminated because they were not related to customer preferences, such as some impractical and imaginative cell phone functions in the future. The 42 queries left are classified as general queries, specific queries, and context queries [27]. The general queries are associated with the upper-level concepts of the ontology, such as customer preferences of different cell phone brands or their series, while the specific queries are associated with lowerlevel concepts of taxonomies, e.g., function and performance, shape features and material attributes. The third category is context queries that can not be easily described except for

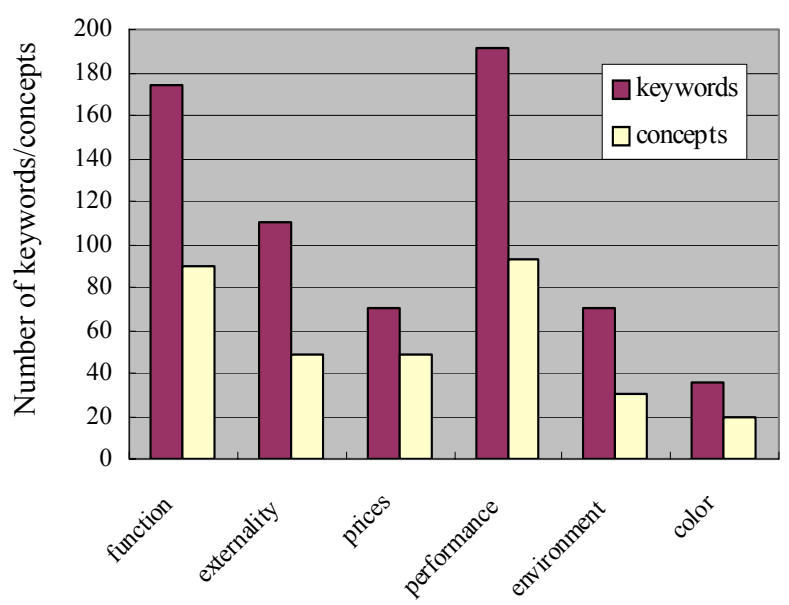

Figure 9 Distribution of keywords/concepts

context expression, in which the customers specify a certain context in order to make the query unambiguous, such as cell phone performance parameters or quantitative indexes. We can use Protégé 3.1 (http://protege.stanford.edu/) to generate domain ontology, in which preference taxonomy was generated as the basis of concept hierarchies. The lexical terms of the concept were modeled as the slot attribute of each concept class. This also supports the domain ontology model in several formats, such as XML, OWL and RDF. The domain ontology model was translated into XML script and input into the system [29]. Ontology concepts are built based on customer preference. Their interrelationships and number of types are statistically calculated and shown in Fig.9.

\section{CONCLUDING REMARKS}

In this paper, we have developed the customer preference ontology, and preference information is extracted to build a preference thesaurus. Ontology-based model is developed for the information extraction. The concept generation and selection of information are based on customer preference ontology. We have shown how the ontology can be used to generate and measure design concepts in customer queries. We have used the preference domain knowledge of cell phone for describing the proposed approach, while the results can be applied to other similar products. Our ontology-based retrieval demonstrates its superiority to keyword-based search techniques by evaluating recall and precision. However, the further research work is needed in many different dimensions and applications of customer preference ontologies.

- As many new technologies are being developed as well as converge with each other, the varieties of product will continue to increase. As a result, design requirements of data will become more complex. Thus the large amount of unstructured and informal design information will also continue to steadily increase, such as engineers' log, product image, nonstandard language descriptions, etc. These texts are less likely to comply with the formal documentation format [32]. At the same time, they are still a part of customer preference knowledge.

- Customer preferences are not static and are indeed changeable. At a particular time, customers show a strong liking for certain product types. But later their preferences perhaps change and they show a liking for another category. Therefore, we would like to build an ontology that is easy to update and can dynamically adapt to customer preference changes. In addition, as time goes on, customer perceptions and product concepts are constantly changing around customer preferences. An automatic analysis approach to keeping abreast of the changes is needed to satisfy with a fast and simple response to customer preferences and changes in the market. This may also entail automating some of the ontology building functions.

In addition to the proposed application, our foundation and approach can also be used from queries of customers in various search engines after preliminary classification. One may have subscription services based on special areas where the market research can transform to an online service function.

\section{ACKNOWLEDGMENTS}

We would like to acknowledge partial support of Product Lifecycle Management (PLM) center at Purdue University and the Center for Advanced Manufacturing (CAM). We also would like to thank anonymous reviewers for their helpful suggestions for this study. 


\section{REFERENCES}

[1] Gerald Zaltman, 2003. How customers think Harvard Business School Press

[2] Sarah Lichtenstein, Paul Slovic, 2006. The construction of preference, Cambridge University Press

[3] John R. Hauser, Duncan Simester, and Birger Wernerfelt, 1994, Customer satisfaction incentives, Marketing Science, Vol. 13, No.4, pp. 327-350.

[4] P M A Desmet, J C O Nrtiz and J P Schoormans, 2008. Product personality in physical interaction, Design Studies, Vol.29, pp. 458-477.

[5] E.F. MacDonald, R. Gonzalez, P.Y. Papalambros, 2009. Preference inconsistency in multidisciplinary design decision making, Journal of Mechanical Design, Vol. 131/ 031009-1.

[6] J Jiao, C H Chen, 2006. Customer requirement management in product development: A review of research issues, Concurrent Engineering, 14(3):173-185.

[7] P Desmet, J V Erp and M Karlsson, 2008. Design \& emotion moves, Cambridge Scholars Publishing.

[8] Griffin, A., Hauser, J. R., 1993. The voice of the customer, Marketing Science, Vol.12, No.1, pp.1-27.

[9] R Mugge, J P L Schoormans, H N J Schifferstein, 2009. Emotional bonding with personalised products, Journal of Engineering Design, 20:5, 467-476.

[10] Li, Z., Raskin, V., and Karthik Ramani, 2008. Developing engineering ontology for information retrieval, Journal of Computing and Information Science in Engineering, Vol.8/011003-1-13.

[11] Claes, F., 1992. A national customer satisfaction barometer: The Swedish experience, Journal of Marketing, Vol.56, pp. 6-21.

[12] Klaus, G. G., Tino, B. L., 2005. Explaining choice option attractiveness by beliefs elicited by the laddering method, Journal of Economic Psychology, Vol.26, pp.223-241.

[13] Thorsten, G., Alexander, R., Isabelle, S., Roediger, V., 2008. Revealing the expectations and preferences of complaining customers by combining the laddering interviewing technique with the Kano model of customer satisaction, Qualitative Market Research: An International Journal, Vol.11, No.4, pp. 400-413.

[14] D'Ambrosio, J. G., and Birmingham, W. P. 1995. Preference-directed design. AI EDAM 9(3), 219-230.

[15] Linoff, G. S., Berry, M. J. A., 2001. Mining the web: Transforming customer data into customer value, John Wiley \& Sons, New York

[16] Yang, Maria C., Wood III, W. H., Cutkosky, M. R., 2005. Design information retrieval: a thesauri-based approach for reuse of informal design information, Engineering with Computers, Vol. 21, pp.177-192.

[17] S Orsborn, J Cagan, P Boatwright, 2009. Quantifying aesthetic form preference in utility function, Journal of Mechanical Design, Vol.131/ 061001-1-10.
[18] Berkowitz, M., 1987. Product shapes as a design innovation strategy, J. Prod. Innovation Manage, Vol. 4, No. 4, pp.274-283.

[19] Iyer N, Jayanti S, Lou K, Kalyanaraman Y, Ramani. Three-dimensional shape searching: state-of-the-art review and future trends Computer-Aided Design, 37 (2005) 509-530.

[20] Gruber T, 1995. Towards principles for the design of ontologies used for knowledge sharing, International Journal of Human-Computer Studies, 43 (5-6), 907-928.

[21] Uschold M and King M, 1995. Towards a methodology for building ontology, IJCAI95 Workshop on Basic Ontological Issues in Knowledge Sharing, Montreal.

[22] Grüninger M and Fox M S, 1995. Methodology for the design and evaluation of ontologies. Proc. Int. Joint Conf. AI Workshop on Basic Ontological Issues in K nowledge Sharing, Montreal.

[23] Orsborn S, Cagan J, Boatwright P, 2009. Quantifying aesthetic form preference in utility function, Journal of Mechanical Design, Vol.131/ 061001-1-10

[24] McCormack J and Cagan J, 2002. Supporting designer's hierarchies through parametric shape recognition, Environ Plann B Plann Des, Vol.29, pp.913-931.

[25] Zeng Y, 2004. Environment-based formulation of design problem, Integrated Design and Process Technologies, Vol. 8, No.4, pp.45-63.

[26] Li Z, Yang MC and Ramani K., 2009. A methodology for engineering ontology acquistion and validation, Artifical Intelligence for Engineering Design, Analysis and Manufacturing, Vol. 23, pp. 37-51.

[27] Khan, L., McLeod, D., Hovy, E., 2004. Retrieval effectiveness of an ontology-based model for information retrieval, Int. J. Very Large Data Base (VLDB), Vol.13, pp.71-85.

[28] Miller, G. A., 1995. Wordnet: a lexical database for English, Communication of the ACM, 38 (11): 39-41.

[29] Lim S C J, Liu Y, Lee W B, 2009. Product analysis and variants derivation based on a semantically annotated product family ontology, ASME IDETC 2009, Aug.30, San Diego, USA.

[30] Gómez-Pérez A, 1998. Knowledge sharing and reuse, The Handbook of Applied Expert Systems, J.Liebowitz, ed., CRC Press, Boca Raton, Fla.

[31] Ahmed S, Kim S and Wallace K M, 2007. A methodology for creating ontologies for engineering design, Journal of Computing and Information Science in Engineering, Vol.7, 132-140.

[32] Castells P, Fernandez M, and Vallet D, 2007. An adaptation of the vector space model for ontology based information retrieval, IEEE Transaction on Knowledge and Data Engineering, 19 (2): 261-272. 